\title{
NECESSARY AND SUFFICIENT CONDITIONS FOR THE REPRESENTATION OF A FUNCTION BY A DOUBLY INFINITE LAPLACE INTEGRAL*
}

\author{
BY D. V. WIDDER
}

1. Introduction. By a doubly infinite Laplace integral we mean the improper Stieltjes integral

$$
\int_{-\infty}^{\infty} e^{-x t} d \alpha(t)
$$

Here $\alpha(t)$ is a function of bounded variation in every finite interval, and

$$
\int_{-\infty}^{\infty} e^{-x t} d \alpha(t)=\lim _{R \rightarrow \infty} \int_{0}^{R} e^{-x t} d \alpha(t)+\lim _{R \rightarrow \infty} \int_{-R}^{0} e^{-x t} d \alpha(t) .
$$

We are concerned in this note with the case in which $\alpha(t)$ is an increasing function. We propose to characterize the class of functions which can be represented by such integrals.

In order to foresee what type of result may be expected we first recall certain properties of the moments of a function on a finite interval. Set

$$
\mu_{n}=\int_{0}^{1} y^{n} d \chi(y), \quad(n=0,1,2, \cdots),
$$

where $\chi(y)$ is a real increasing function. It was proved by $F$. Hausdorff $\nmid$ that equations (1) have a solution $\chi(y)$ if and only if the sequence $\left\{\mu_{n}\right\}$ is completely monotonic,

$$
(-1)^{k} \Delta^{k} \mu_{n} \geqq 0, \quad(n, k=0,1,2, \cdots) .
$$

If the integer $n$ in (1) is replaced by a continuous variable $x$, we obtain

$$
\mu(x)=\int_{0}^{1} y^{x} d \chi(y),
$$

* Presented to the Society, December 27, 1933.

$\dagger$ F. Hausdorff, Momentprobleme für ein endliches Intervall, Mathematische Zeitschrift, vol. 16 (1923), p. 220. 
or

$$
\mu(x)=\int_{0}^{\infty} e^{-x t} d \alpha(t),
$$

if we set $y=e^{-t}$ and $\alpha(t)=-\chi\left(e^{-t}\right)$. We should expect conditions (2) to be replaced by

$$
(-1)^{k} \mu^{(k)}(x) \geqq 0, \quad(x>0 ; k=0,1,2, \cdots) .
$$

The conjecture arrived at in this way has proved to be correct. $S$. Bernstein,* the author, $\dagger$ and others have shown that (4) is necessary and sufficient that the integral equation (3) should have an increasing solution $\alpha(t)$.

Since analogy leads to a correct conjecture regarding (3), it is natural to hope that a similar analogy will be equally successful for the doubly infinite Laplace integral. Clearly we must now consider the Stieltjes moment problem,

$$
\mu_{n}=\int_{0}^{\infty} y^{n} d \chi(y), \quad(n=0,1,2, \cdots) .
$$

It is known $\ddagger$ that equations (5) have an increasing solution $\chi(y)$ if and only if the quadratic forms

$$
\sum_{i=0}^{n} \sum_{j=0}^{n} \mu_{i+j} \xi_{i} \xi_{j}, \sum_{i=0}^{n} \sum_{j=0}^{n} \mu_{i+j+1} \xi_{i} \xi_{j}, \quad(n=0,1,2, \cdots),
$$

are positive (definite or semi-definite). Making the same transformations as those we performed on (1), we obtain

$$
\mu(x)=\int_{-\infty}^{\infty} e^{-x t} d \alpha(t), \quad \alpha(t)=-\chi\left(e^{-t}\right) .
$$

We should expect the quadratic forms (6) to coalesce into a single double integral of the form

* S. Bernstein, Sur les fonctions absolument monotones, Acta Mathematica, vol. 52 (1929), p. 1.

$\dagger \mathrm{D}$. V. Widder, Necessary and sufficient conditions for the representation of a function as a Laplace integral. Transactions of this Society, vol. 33 (1931), p. 851 .

¥ See, for example, O. Perron, Die Lehre von den Kettenbrïchen, second edition, 1922, Chapter 9. 


$$
\iint \mu(x+y) \xi(x) \xi(y) d x d y
$$

which would be required to be positive for all functions $\xi(x)$. We shall show that this condition, when made sufficiently precise, is necessary and sufficient for the representation of the function $\mu(x)$ in the form (7) with an increasing function $\alpha(t)$, and consequently carries with it the analyticity of $\mu(x)$ in a vertical strip of the complex plane. The proof is made by use of certain results of J. Mercer, H. Hamburger, and S. Bernstein. These we state as lemmas with appropriate references. It is of interest to compare the present result with one of M. Mathias* and S. Bochner $\dagger$ concerning the Fourier transform. We also call attention to a related result of $\mathrm{S}$. Bernstein $\ddagger$ concerning "exponentially convex functions".

2. The Lemmas. We first introduce several definitions.

Definition 1. If the function $K(s, t)$ is continuous in the closed square $a \leqq s \leqq b, a \leqq t \leqq b$, and if

$$
\int_{a}^{b} \int_{a}^{b} K(s, t) \xi(s) \xi(t) d s d t \geqq 0
$$

for all functions $\xi(s)$ continuous in the interval $a \leqq s \leqq b$, then $K(s, t)$ is said to be a kernel of positive type in the square.

Definition 2. The function $K(s, t)$ is of positive type in the open square $a<s<b, a<t<b$ if it is of positive type in every closed square $a^{\prime} \leqq s \leqq b^{\prime}, a^{\prime} \leqq t \leqq b^{\prime}$ for which $a<a^{\prime}<b^{\prime}<b$.

LEMma 1. (Mercer.§) A necessary and sufficient condition that

* M. Mathias, Über positive Fourier-Integrale, Mathematische Zeitschrift, vol. 16 (1923), p. 101.

$\dagger$ S. Bochner, Vorlesungen über Fouriersche Integrale, 1932, p. 76.

$\ddagger \mathrm{S}$. Bernstein, loc. cit., p. 60. However, the result stated in the article cited is open to question. If we denote by $\phi(x)$ the function of G. Hamel (Mathematische Annalen, vol. 60 (1905), p. 459) which satisfies the functional equation $\phi(x+y)=\phi(x)+\phi(y)$, we find that $\exp \phi(x)$ is exponentially convex. But such a function can not have the representation (7) since it is not even continuous, much less analytic. I am indebted to Hassler Whitney for calling my attention to the function of Hamel.

$\S \mathrm{J}$. Mercer, Functions of positive and negative type, and their connection with the theory of integral equations, Transactions of the Royal Society of London, (A), vol. 209 (1909), p. 415. 
a continuous function $K(s, t)$ be of positive type in the square $a^{\prime} \leqq s \leqq b^{\prime}, a^{\prime} \leqq t \leqq b^{\prime}$ is that the quadratic forms

$$
\sum_{i=0}^{n} \sum_{j=0}^{n} K\left(s_{i}, s_{j}\right) \xi_{i} \xi_{j}, \quad(n=0,1,2, \cdots),
$$

be positive (definite or semi-definite) for all values of $s_{i}$ and $s_{j}$ in the interval $a \leqq s \leqq b$.

LEMma 2. (Bernstein.*) If the function $f(x)$ is continuous in $a<x<b$, and if

$$
\Delta^{(2 n)} f(c)=\sum_{k=0}^{2 n}(-1)^{k}\left(\begin{array}{c}
2 n \\
k
\end{array}\right) f(c+k \delta) \geqq 0, \quad(n=0,1,2, \cdots),
$$

for every choice of $c$ and $\delta$ such that

$$
a<c<c+\delta<c+2 \delta<\cdots<c+2 n \delta<b,
$$

then $f(x)$ is analytic in $a<x<b$.

LEMMA 3. (Hamburger. $\dagger$ ) If $f(x)$ is analytic in the interval $a<x<b$, and if the quadratic forms

$$
\sum_{i=0}^{n} \sum_{j=0}^{n} f^{(i+j)}(c) \xi_{i} \xi_{j}, \quad(n=0,1,2, \cdots),
$$

are positive (definite or semi-definite) for a single value of $c$ in $a<x<b$, then

$$
f(x)=\int_{-\infty}^{\infty} e^{-x t} d \alpha(t)
$$

where $\alpha(t)$ is a non-decreasing function, and the integral converges for $a<x<b$.

3. The Theorem. By use of the above lemmas we now prove the following theorem.

* S. Bernstein, Leçons sur les Propriêtés Extrémales des Fonctions Analytiques d'une Variable Reelle, 1926, pp. 190-197. The proof there given needs slight modification to cover the case in hand. We call particular attention to the fact that the assumed continuity of $f(x)$ in Lemma 2 is not a redundant condition.

$\dagger \mathrm{H}$. Hamburger, Bemerkungen zu einer Fragestellung des Herrn Polya, Mathematische Zeitschrift, vol. 7 (1919), p. 302. The theorem is there stated for the case in which the forms (8) are positive definite, but the proof holds equally well when they are positive semi-definite. 
TheOREM. A necessary and sufficient condition that the function $f(x)$ can be represented in the form

$$
f(x)=\int_{-\infty}^{\infty} e^{-x t} d \alpha(t),
$$

where $\alpha(t)$ is non-decreasing and the integral converges for $a<x<b$, is that the kernel $f(s+t)$ be continuous and of positive type in the square $a<2 s<b, a<2 t<b$.

We shall prove first the necessity of the condition. Let $f(x)$ have the representation (9). Let $a<a^{\prime}<b^{\prime}<b$. Then the kernel $f(s+t)$ is of positive type in the square $a^{\prime} \leqq 2 s \leqq b^{\prime}, a^{\prime} \leqq 2 t \leqq b^{\prime}$. For, let $\xi(t)$ be an arbitrary continuous function in the interval $a^{\prime} \leqq 2 t \leqq b^{\prime}$. Then

$$
\begin{aligned}
& \int_{a^{\prime} / 2}^{b^{\prime} / 2} \int_{a^{\prime} / 2}^{b^{\prime} / 2} f(s+t) \xi(s) \xi(t) d s d t \\
= & \int_{a^{\prime} / 2}^{b^{\prime} / 2} \int_{a^{\prime} / 2}^{b^{\prime} / 2} \xi(s) \xi(t) d s d t \int_{-\infty}^{\infty} e^{-(s+t) u} d \alpha(u) \\
= & \int_{-\infty}^{\infty}\left[\int_{a^{\prime} / 2}^{b^{\prime} / 2} e^{-s u} \xi(s) d s\right]^{2} d \alpha(u) \geqq 0 .
\end{aligned}
$$

The change in the order of integration is easily justified since the integral (9) converges uniformly in $a^{\prime} \leqq x \leqq b^{\prime}$.

To prove the sufficiency of the condition we first apply Lemma 1 . Since $f(s+t)$ is by hypothesis of positive type in the square $a^{\prime} \leqq 2 s \leqq b^{\prime}, a^{\prime} \leqq 2 t \leqq b^{\prime}$, it follows (by setting $s_{0}=c / 2$, $\left.s_{1}=(c / 2)+\delta, \cdots, s_{n}=(c / 2)+n \delta\right)$ that the quadratic forms

$$
\sum_{i=0}^{n} \sum_{j=0}^{n} f(c+i \delta+j \delta) \xi_{i} \xi_{j}, \quad(n=0,1,2, \cdots),
$$

are positive, provided

$$
a^{\prime} \leqq c<c+\delta<c+2 \delta<\cdots<c+2 n \delta \leqq b^{\prime} .
$$

In particular, if we set

$$
\xi_{i}=\sum_{k=i}^{n}(-1)^{k+1}\left(\begin{array}{c}
k \\
i
\end{array}\right) \eta_{k}, \quad(i=0,1,2, \cdots, n),
$$


the forms (10) become

$$
\sum_{i=0}^{n} \sum_{j=c}^{n} \Delta^{(i+j)} f(c) \eta_{i} \eta_{j}
$$

If $\eta_{0}=\eta_{1}=\cdots=\eta_{n-1}=0, \eta_{n}=1$, we see that

$$
\Delta^{(2 n)} f(c) \geqq 0 .
$$

Since $f(x)$ is continuous by hypothesis, we may apply Lemma 2 and deduce that $f(x)$ is analytic in $a<x<b$. In (11) replace $\eta_{i}$ by $\eta_{i} / \delta$ and let $\delta$ approach zero. We thus obtain

$$
\sum_{i=0}^{n} \sum_{j=0}^{n} f^{(i+j)}(c) \eta_{i} \eta_{j} \geqq 0,
$$

and by Lemma 3 , the function $f(x)$ has the form (9). This completes the proof of the theorem.

HARVARD UNIVERSITY

\title{
ARITHMETIC AND IDEAL THEORY OF ABSTRACT MULTIPLICATION*
}

\author{
BY A. H. CLIFFORD
}

If we are given a ring $R$ we may be called upon to answer the following two questions.

1. Is every element of $R$ uniquely decomposable into prime elements?

2. If not can we introduce ideal elements into $R$ such that the resulting system has this property?

Since these questions can be put in terms involving only the operation of multiplication, it is natural to attempt a solution in the same terms. We start, therefore, with a group-like system in which multiplication only is defined, namely a class $S$ satisfying the following postulates:

* A statement of definitions and results of a thesis done under Professors E. T. Bell and Morgan Ward at the California Institute of Technology.

(Added in proof.) I find that ovoid ideals were first discovered by I. Arnold, Ideale in kommutativen Halbgruppen, Recueil Mathématique, Moscou, vol. 36 (1929), pp. 401-407. Arnold proves Theorem 4 for regular ova (which he calls commutative semi-groups), with a slightly different normal ideal arithmetic. 